\title{
Ensilaje de naranjas enteras (Citrus sinensis) como suplemento para alimentación de rumiantes
}

\author{
Edna Paola Melo-Camacho $\mathbb{D}^{1}$, Jaime Alberto Bermúdez-Loaiza (iD ${ }^{1}$, Julián Estrada- \\ Álvarez (1D ${ }^{2}$ \\ ${ }^{1}$ Maestría en Sistemas de Producción Agropecuaria, Facultad de Ciencias \\ Agropecuarias, Universidad de Caldas, Manizales, Caldas, Colombia. \\ ${ }^{2}$ Docente, Departamento de Producción Agropecuaria, Universidad de Caldas, \\ Manizales, Caldas, Colombia.
}

jestrada@ucaldas.edu.co

\begin{abstract}
Recibido: 28 de Octubre de 2016, Aprobado: 8 de Febrero de 2017, Actualizado: 20 de Junio de 2017
\end{abstract}

DOI: 10.17151/vetzo.2017.11.1.3

RESUMEN: Se han utilizado residuos de naranja (cáscaras) en alimentación no convencional para rumiantes, a través de conservación mediante ensilaje, método de fermentación de materiales celulósicos, que convierte los carbohidratos solubles, en ácidos orgánicos y agua, permitiendo conservar el producto; pero no se han reportado ensayos de ensilaje de frutos enteros de naranja, de manera que se pueda conservar el valor nutritivo del jugo y que no se genere el impacto negativo debido a los efluentes. En el presente estudio se ensiló una tonelada de naranja entera en canecas plásticas y se determinó $\mathrm{pH}$, humedad, materia seca, nitrógeno total, grasa total, fibra bruta, cenizas totales, fósforo, potasio, calcio, magnesio, sodio, hierro, cobre, manganeso, zinc y energía bruta, a los 22, 29 y 45 días de ensilado, analizando las variables de calidad composicional a través del tiempo, se concluyó que es factible ensilar frutos enteros de naranja, para la alimentación de rumiantes sin ofrecer efectos negativos de lixiviación (pérdidas de nutrientes) y resaltando su aporte energético para el animal.

Palabras clave: gestión de residuos agrícolas, naranja entera, nutrición, suplementación

\section{Silage of whole oranges (Citrus sinensis) as supplement for ruminants feeding}

ABSTRACT: The orange residues (shells) have been used in nonconventional feed for ruminants, through their conservation by means of silage, fermentation method of cellulosic materials, which converts soluble carbohydrates into organic acids and water, allowing the product to be conserved; however, no trials of silage of whole fruits of orange have been reported, so that the nutritive value of the juice can be conserved without generating the negative impact of the effluents. In this study a ton is ensiled of whole orange in plastic containers and was determined $\mathrm{pH}$, humidity, dry matter, total nitrogen, protein gross, total fat, crude fiber, total ashes, phosphorus, potassium; calcium, magnesium, sodium, iron, copper, manganese, zinc and crude energy, at 22, 29 and 45 days of anaerobic conditions. Analyzing the compositional quality variables over time, it was concluded that it is feasible to silage 
whole fruits of orange, to feeding ruminants without offering negative effects of leaching (losses of nutrients) and highlighting them energy contribution for the animal.

Key words: agricultural waste management, nutrition, supplementation, whole orange

\section{Introducción}

Colombia posee condiciones favorables para cultivar cítricos (Aguilar et al., 2012), en el 2013 se estimaban 58.925 ha sembradas (56\% en naranjas), con una producción de 749.536 t/año (DANE, 2013), generando el 24\% de empleos directos generados por el sector frutales. Según CitriCaldas (2014), el Eje Cafetero contaba con 2.881 ha sembradas en cítricos (78\% naranjas) y los mismos productores reportaron 1.794 cabezas bovinas para suplementar. Nuestra producción es estacional, principal (de mayo-julio) y mitaca (de octubre-diciembre) (ACCPC, 2000; Martínez et al., 2005); esta sobreproducción estacional incrementa la oferta y disminuye el precio.

Los cítricos son de gran importancia en la industria alimentaria y cosmética (Lante \& Tinello, 2015) por su generación de residuos. Marín et al. (2007) estimaron 20 millones de toneladas de residuos agroindustriales cítricos (RAC) al año, a partir de la industria de jugos (cáscara y pulpa prensada), fruta desechada (en mal estado) y descartada por las regulaciones que limitan la producción (Ruiz \& Flotats, 2014); la cáscara representa del 45 al 60\% del peso total de la fruta (Rincón et al., 2005); los altos costos de eliminación de RAC en el mundo están impidiendo que las empresas sigan siendo competitivas (Bampidis \& Robinson, 2006).

Martínez et al. (2008) aseguran que los RAC pueden convertirse en alimentos no convencionales para bovinos; pero, no se conoce su uso como frutos enteros (FE) para alimentación animal (Volanis et al., 2004); los RAC utilizados como alimentos son: pulpa fresca, ensilaje, pulpa deshidratada, harina, melazas, licor de la cáscara y lodos activados (Bampidis \& Robinson, 2006). Gran parte de la pulpa de cítricos (PC) fresca se pierde por la dificultad de consumirla rápidamente, a través del ensilaje, de manera que permita ser conservada, sin embargo, produce altos efluentes, disminuyendo su valor nutritivo e impactando el medio ambiente (Caparra et al., 2007).

El RAC posee una humedad del 84,7\% (Piquer et al., 2009), proteína bruta 7,6\% y fibra bruta 14,3\%; no poseen lignina en su estructura, sólo celulosa que es altamente degradable en el rumen (Ojeda et al., 2008); los RAC poseen sustancias químicas de interés industrial y para la alimentación animal, entre otros (Londoño et al., 2012). Los aceites esenciales cítricos (AEC) son sustancias lipofílicas, presentes en el flavedo principalmente, que algunas veces son usados como insecticidas químicos (Isman, 2000). Estudios previos demostraron que los AEC no dificultan la fermentación del ensilaje y representan bajo riesgo para humanos y animales (Fisher \& Phillips, 2008).

El ensilaje es un método de fermentación anaeróbica, donde bacterias ácido lácticas (BAL) convierten los carbohidratos solubles, en ácidos orgánicos y agua; disminuyendo 
el pH para conservar el material (Gollop et al., 2005); los RAC pueden ser ensilados como alimentos para rumiantes sin ofrecer efectos negativos (Volanis et al., 2004).

El ensilaje pasa por seis fases (Driehuis \& Oude, 2000): 1. Fase enzimática: después del corte, las enzimas del vegetal hidrolizan parte de las proteínas verdaderas, del almidón y de la hemicelulosa, causando pérdidas de distinto orden y generando azúcares que serán utilizados durante la fermentación láctica (Muck, 2013). 2. Fase aeróbica: genera una respiración residual (Dunière et al., 2013), el oxígeno atrapado en la masa ensilada se reduce rápidamente por la actividad respiratoria del material vegetal y microorganismos aerobios facultativos (Driehuis \& Oude, 2000). 3. Fase anaeróbica: agotado el O2 es reemplazado por CO2 generando anaerobiosis, así las BAL, favorecidas por la difusión de los jugos celulares (azúcares) le sirven como fuente energética. 4. Fase de fermentación o acidificación: se inicia cuando las BAL (homo y heterofermentadoras) dominan la fermentación, disminuyendo rápido el pH debido a la acumulación de ácido láctico y acético (Driehuis \& Oude, 2000). 5. Fase de almacenamiento o estabilización: el número de microorganismos viables disminuye (Driehuis \& Oude, 2000) y se generan pocos cambios en el ensilaje (Driehuis \& Oude, 2000; Dunière et al., 2013). 6. Fase de deterioro aeróbico: iniciada cuando el ensilaje queda expuesto al aire, provocando un período de oxidación de los ácidos orgánicos conservantes, incrementa el pH e inicia la proliferación de organismos anaeróbicos indeseables (levaduras ácido tolerantes) (Driehuis \& Oude, 2000).

Los inoculantes BAL (homo y heterofermentativas) determinan la conservación, garantizando poblaciones que inhiben la degradación de proteínas (Wang et al., 2009; Gollop et al., 2005) y permiten la acumulación rápida de ácidos orgánicos (ácido láctico), responsable de la disminución rápida del pH (Dunière et al., 2013), además reduce la supervivencia de levaduras (Driehuis et al., 1999) que son indeseables, por la producción de alcoholes.

El objetivo principal de este proceso fue a través del ensilaje encontrar alternativas de alimentación para consumo animal, mejorando así la sostenibilidad económica y ambiental de los sistemas de producción (Dunière et al., 2013).

\section{Materiales y Métodos}

El experimento se desarrolló en la granja La Cruz de la Universidad de Caldas (vereda Las Margaritas, municipio de Anserma - Caldas), ubicada a 75²3'19.8' longitud oeste y $5^{\circ} 07^{\prime} 35.0^{\prime}$ latitud norte, a $1053 \mathrm{msnm}$, temperatura media de $24^{\circ} \mathrm{C}$, humedad relativa del 75\%, precipitación anual media de 1076 mm, zona de vida Bosque seco Tropical (BsT).

Los frutos de naranja (variedad Valencia) fueron obtenidos de las fincas El Tamboral y El Ruby, ubicadas a 75³6'35.61' longitud oeste y $5^{\circ} 06^{\prime} 42.06^{\prime}$ latitud norte y a 75³6'763' longitud oeste y $5^{\circ} 07^{\prime} 324^{\prime}$ latitud norte, respectivamente (vereda La Cristalina, municipio de Manizales), a $977 \mathrm{msnm}$, temperatura media de $25^{\circ} \mathrm{C}$, humedad relativa del 80\%, precipitación anual media de $2100 \mathrm{~mm}$, zona de vida selva muy 
húmeda Montaña (smh-M); los frutos fueron de tercera calidad con diámetro ecuatorial inferior a 53 mm, grado de maduración pintón, enteros y con buen estado general.

Para el ensilaje de naranja entera se utilizaron canecas plásticas con capacidad para 45 kg con tapa hermética y válvula de seguridad y 10 g de Sil all ${ }^{\circledR}$ diluido en 2 L de agua destilada (10g/Ton de naranja). Se colocaron naranjas hasta la mitad de cada caneca y se roció Sil all®, luego se terminó de llenar la caneca y se volvió a rociar con el inóculo. Se cubrió la caneca con la tapa hermética y se reforzó con cinta de policloruro de vinilo (PVC) marca Truper ${ }^{\circledR}$; el aire fue extraído a través de la válvula de seguridad con una aspiradora Karcher ${ }^{\circledR}$ industrial durante 20 segundos aproximadamente, se almacenaron y fueron dejadas en estas condiciones durante 21 días; transcurrido este tiempo se tomaron muestras del ensilaje a los 22, 29 y 45 días de los tres tratamientos (t1, t2 y t3), respectivamente, y se determinó: $\mathrm{pH}$, humedad $(\mathrm{H})$, materia seca (MS), nitrógeno total (NT), proteína bruta (PB), grasa total (GT), fibra bruta (FB), cenizas totales (CT), fósforo $(\mathrm{P})$, potasio $(\mathrm{K})$; calcio $(\mathrm{Ca})$, magnesio $(\mathrm{Mg})$, sodio $(\mathrm{Na})$, hierro $(\mathrm{Fe})$, cobre $(\mathrm{Cu})$, manganeso $(\mathrm{Mn})$, zinc $(\mathrm{Zn})$ y energía bruta $(\mathrm{EB})$.

Métodos y equipos utilizados en laboratorio son referenciados en la tabla 1. 
Tabla 1. Pruebas de laboratorio.

\begin{tabular}{|c|c|c|}
\hline $\begin{array}{l}\text { Prueba de } \\
\text { Laboratorio }\end{array}$ & Método & Equipo \\
\hline $\mathrm{pH}$ & Potenciométrico & $\begin{array}{l}\text { pHmetro Marca Hanna } \otimes \text {, } \\
\text { referencia Hi } 4522 \text {. }\end{array}$ \\
\hline H y MS & $\begin{array}{l}\text { Gravimétrico (AOAC, } \\
\text { 2012) }\end{array}$ & $\begin{array}{l}\text { Estufa de aire circulante Marca } \\
\text { Thelco }{ }^{\odot} \text {, Modelo } 6557 \text {, Serie: } \\
60278 .\end{array}$ \\
\hline CT & $\begin{array}{l}\text { Calcinación (AOAC, } \\
2012)\end{array}$ & $\begin{array}{l}\text { Mufla Marca Furnace }{ }^{\odot} \text {, Modelo } \\
62700 \text {. }\end{array}$ \\
\hline GT & Solvent (AOAC, 2012) & $\begin{array}{l}\text { Extractor de solvente, Marca: Velp } \\
\text { Scientifica }^{\circ} \text {, Serie: } 148 \text {. }\end{array}$ \\
\hline FB & $\begin{array}{l}\text { Digestión ácido } \\
\text { alcalina según } \\
\text { "Weende" }\end{array}$ & $\begin{array}{l}\text { Extractor de Fibra, Marca: Velp } \\
\text { Scientifica }{ }^{\ominus} \text {, Serie: FIWE. }\end{array}$ \\
\hline $\mathrm{N}$ & $\begin{array}{l}\text { Kjeldahl (Kjeldahl, } \\
\text { 1883). } \\
\text { Se utilizó 6,25 como } \\
\text { factor de conversión } \\
\text { para PB. }\end{array}$ & $\begin{array}{l}\text { Equipo de digestión Marca Buchi }{ }^{\odot} \\
\text { Referencia K-436 y destilador } \\
\text { Buchi Referencia B-324, }\end{array}$ \\
\hline$P$ & Colorimétrico & $\begin{array}{l}\text { Espectrofotómetro Ultra Violeta } \\
\text { Visible, Dynámica }{ }^{\circledR} \text { Referencia } \\
\text { HALO RB-10 y se leyó la } \\
\text { absorbancia a } 660 \mathrm{~nm} \text {. }\end{array}$ \\
\hline $\begin{array}{c}\mathrm{Ca} \\
\mathrm{Mg} \\
\mathrm{Fe} \\
\mathrm{Mn} \\
\mathrm{Zn} \\
\mathrm{Cu}\end{array}$ & Absorción Atómica & $\begin{array}{l}\text { Espectrofotómetro de Absorción } \\
\text { Atómica, Thermo Elemental }{ }^{\odot} 969 \\
\text { SOLAAR con lámparas de cátodo } \\
\text { hueco Thermo Scientific }{ }^{\odot} \\
\text { específicas para cada elemento. } \\
\text { Leídos a las siguientes longitudes } \\
\text { de onda: } \\
\text { Ca } 422,3 \mathrm{~nm}, \mathrm{Mg} 285,7 \mathrm{~nm}, \mathrm{~K} \\
766,5 \mathrm{~nm}, \mathrm{Fe} 248,3 \mathrm{~nm}, \mathrm{Mn} 279,5 \\
\mathrm{~nm}, \mathrm{Zn} 213,9 \mathrm{~nm}, \mathrm{Cu} 324,8 \mathrm{~nm} \text {. }\end{array}$ \\
\hline EB & Calorímetro & $\begin{array}{l}\text { Calorímetro Marca Parr } \\
\text { Referencia } 6200 \text { y bomba de agua } \\
\text { Walter Handling System, Marca } \\
\text { Parr }{ }^{\odot} \text {. }\end{array}$ \\
\hline
\end{tabular}

AOAC, (2012). Official methods of analysis of AOAC international $19^{\text {th }}$ edition. 
Los resultados de las pruebas de laboratorio fueron analizados estadísticamente con el programa $R \AA$, realizando un diseño unifactorial con tres repeticiones, en donde se determinó las diferencias en el ensilaje a través del tiempo (t1, t2 y t3), indicando que hubo diferencia significativa para $\mathrm{pH}$, Ca y $\mathrm{Cu}$; posteriormente, se realizó prueba de Tukey para las variables mencionadas.

\section{Resultados y Discusión}

Se realizó un estudio previo que utilizó microsilos de 2,5 kg, para determinar la viabilidad del ensilaje de FEN, indicado en la tabla 2. Comparando los datos obtenidos en este ensayo se encontró que las variables pH y MS de FEN ensilados disminuyeron respecto a FEN frescos, estos resultados concuerdan con los encontrados por Volanis et al. (2004), cuando evaluaron antes y después de ensilar mezclas de RAC con otros subproductos; el descenso en el $\mathrm{pH}$ se asocia principalmente a la producción de ácido láctico que favorece la calidad del ensilaje (Holzer et al., 2003); la disminución de MS puede ser esperada y atribuida a la producción de gas; las demás variables evaluadas aumentaron a pesar del descenso de la MS, efecto similar fue encontrado por Volanis et al. (2004); sin embargo, hay que resaltar que la literatura no reporta estudios sobre el valor nutricional de FEN ensilados, tampoco comparaciones en el tiempo de ensilaje con respecto a su composición química. Niveles de EB que presenta el ensilaje de FEN en los diferentes tiempos oscila entre de 3,4-3,9 Mcal/kg de MS, que lo clasifican como un ensilaje de alta calidad energética, estudios de RAC indican que pueden ser utilizados en alimentación animal como una dieta de alto potencial de energía que apoya el crecimiento y la lactancia, con menos efectos negativos sobre la fermentación del rumen que alimentos ricos en almidón (Piquer et al., 2009; Bampidis \& Robinson, 2006), esto debido a que son una fuente de energía de pectina que causa poca o ninguna disminución del pH del rumen, aumenta la proporción de ácido acético y disminuye la proporción de ácido propiónico (Bampidis \& Robinson, 2006). 
Tabla 2: Rangos de variables medidas en un ensayo previo de ensilaje en microsilos de $2,5 \mathrm{~kg}$

\begin{tabular}{|l|c|c|}
\hline Variable & Naranjas enteras frescas & $\begin{array}{c}\text { Naranjas enteras } \\
\text { ensiladas }\end{array}$ \\
\hline pH & $3,71-3,78$ & $3,3-4,2$ \\
\hline MS & $18,4-20 \%$ & $15,3-18,2 \%$ \\
\hline NT & $1,1-1,4 \%$ & $1,3-1,6 \%$ \\
\hline PB & $6,9-8,6 \%$ & $8,7-10 \%$ \\
\hline GT & $1,6-2,7 \%$ & $1-4,1 \%$ \\
\hline FB & $6-8,2 \%$ & $7,3-11,9 \%$ \\
\hline CT & $2,6-3,3 \%$ & $3,7-4,7 \%$ \\
\hline P & $0,04-0,09 \%$ & $0,08-0,1 \%$ \\
\hline $\mathrm{K}$ & $0,3-0,7 \%$ & $0,9-1,3 \%$ \\
\hline $\mathrm{Ca}$ & $0,3-0,6 \%$ & $0,4-0,7 \%$ \\
\hline $\mathrm{Mg}$ & $0,04-0,07 \%$ & $0,06-0,1 \%$ \\
\hline $\mathrm{Na}$ & $0-0,09 \%$ & $0,02-0,07 \%$ \\
\hline $\mathrm{Fe}$ & $33,5-230,8 \mathrm{mg} / \mathrm{kg}$ & $42,4-421,2 \mathrm{mg} / \mathrm{kg}$ \\
\hline $\mathrm{Cu}$ & $2,3-3,6 \mathrm{mg} / \mathrm{kg}$ & $7,6-20,7 \mathrm{mg} / \mathrm{kg}$ \\
\hline $\mathrm{Mn}$ & $0 \mathrm{mg} / \mathrm{kg}$ & $3,8-12,2 \mathrm{mg} / \mathrm{kg}$ \\
\hline $\mathrm{Zn}$ & $7,5-21,6 \mathrm{mg} / \mathrm{kg}$ & $7,7-15,9 \mathrm{mg} / \mathrm{kg}$ \\
\hline $\mathrm{EB}$ & No evaluado & $3,4-3,9 \mathrm{Mcal}$ \\
\hline
\end{tabular}

A partir de estos resultados y determinando la viabilidad de los microsilos de FEN, se procedió a evaluar el ensilaje de 1 Ton de naranja entera en canecas plásticas.

Ensilaje de FEN en campo (ensilaje de 1 Ton de naranja entera fresca).

A los 21 días de ensilados los FEN presentan buenas condiciones de fermentación, debido a que poseian olor característico del ensilaje de buena calidad, ninguno de los tratamientos presentó olor a putrefacto, se visualizó una reducción de MS en las naranjas enteras, posiblemente debido a la fermentación.

La variable $\mathrm{pH}$ presentó rangos entre 3,3 y 4,2 para los tratamientos como se observa en la figura 1; los resultados coinciden con los reportados por Volanis et al. (2004; 2006), donde se tomaron muestras de $\mathrm{pH}$ antes y después de ensilar, aunque los rangos son más altos, debido a la mezcla de otros subproductos con RAC, los descensos en el pH después del ensilaje son notorios; un $\mathrm{pH}$ inferior a 4 en el ensilaje es indicativo de inocuidad del material (Gollop et al., 2005; Dunière et al., 2013); el efecto conservante del ensilaje se debe principalmente a la producción de ácido láctico, que disminuye el pH y éste a su vez disminuye el potencial de óxido-reducción que posee el material, además, se inhiben los microorganismos y su consumo de nutrientes esenciales (Holzer et al., 2003); en otro estudio realizado por Bermúdez-Loaiza et al., (2015) se evaluó el consumo y la producción bovina, alimentando a 13 vacas lactantes con este ensilaje, arrojando como resultados que las naranjas enteras ensiladas son un producto apto para consumo animal; este resultado es el esperado en procesos bien elaborados de ensilaje, 
debido a la rápida colonización del material por la inoculación con BAL (Dunière et al., 2013), el factor del tiempo debe resaltarse, ya que en la medición a los 29 y 45 días (t2 y t3) el $\mathrm{pH}$ se mantuvo en el mismo rango, indicando que el ensilaje de FEN ha ingresado a la fase de estabilización.

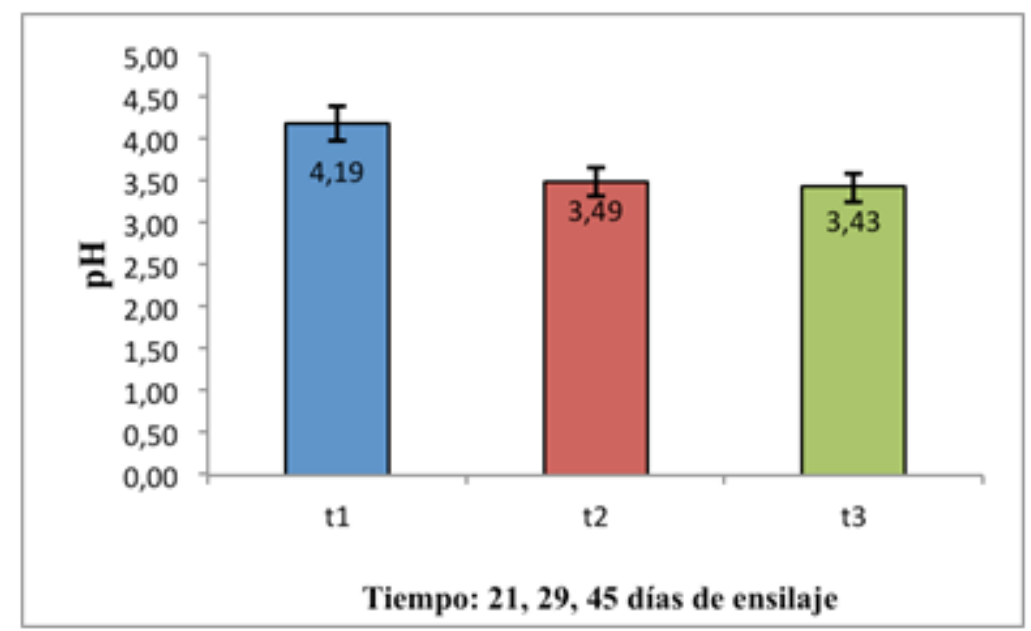

Figura 1. Comportamiento del pH en diferentes tiempos de ensilaje.

La significancia entre los momentos fue de $\mathrm{P}=0,0002(<0,0001$ entre momentos) y Tukey indica que t2 y t3 son iguales entre sí, pero diferentes de t1.

En los análisis de calidad composicional de los ensilajes, no se encontraron diferencias significativas entre tratamientos para CT, GT, FB, N, P, Mg, Fe, Mn, Zn y EB. Solo hubo diferencias para $\mathrm{Ca}$ y $\mathrm{Cu}$. La variable Ca presentó rangos entre 0,4 y 0,7\%, este comportamiento a través del tiempo se observa en la figura 2, el rango de Ca es menor al reportado por Volanis et al., en 2004 que fue de 0,97\% de Ca después del ensilaje; pero en este estudio, los autores no tomaron muestras en varios momentos del ensilaje, sólo antes y después; estas diferencias pueden ser explicadas por la variabilidad genética del material (Turra et al., 2011); el hecho que en este estudio el Ca esté en proporciones similares a los 22 y 29 días y que aumente a los 45 días, se puede deber a la apoptosis en animales, plantas y microbios (Fang et al., 2009), a nivel celular, generando oscilaciones de Ca, permitiendo la entrada masiva de Ca que provoca su muerte (Lobet et al., 2015), aunque algunos autores consideran que este apoptosis no es pertinente en bacterias (Hacker, 2013), mientras otros sí la aceptan e indican que el aumento de iones de Ca inducen la destrucción de bacterias no patogénicas (Trimble \& Grinstein, 2007). Es preciso tener en cuenta que estudios de oscilación de minerales en ensilaje no han sido reportados y estos podrían determinar disponibilidad de algunos elementos en momentos específicos. Para el caso particular del ensilaje de FEN se presentó mayor contenido de $\mathrm{Ca}$ a los 45 días de ensilado. 


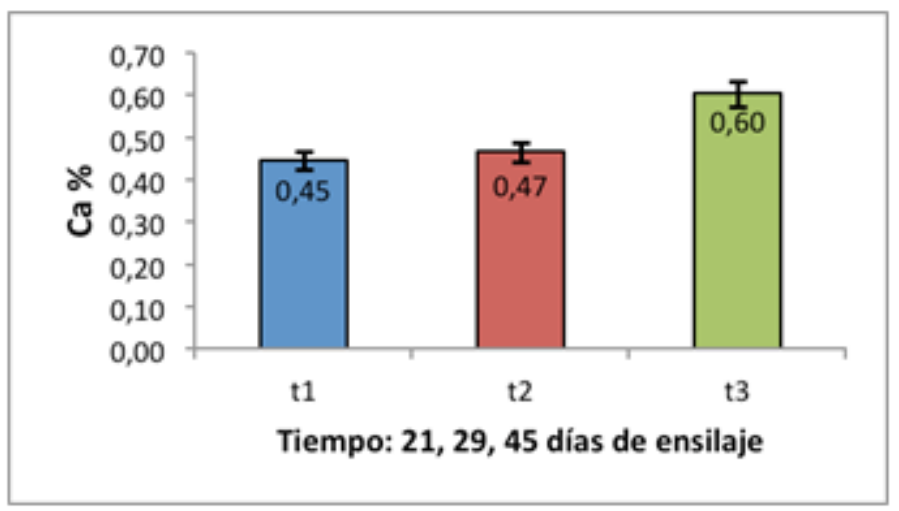

Figura 2. Comportamiento del Ca en diferentes tiempos de ensilaje.

La significancia entre los momentos fue de $\mathrm{P}=0,0166$ (0,00147 y 0,0307 entre repeticiones y momentos respectivamente) y Tukey indica que t1 son iguales t2, pero, diferentes de $\mathrm{t} 3$.

La variable $\mathrm{Cu}$ presentó un incremento notable a los 29 días de ensilaje, este elemento hace parte activa de las bacterias, en el transporte de electrones para la obtención de ATP, por medio de la proteína Citocromo $c$, proceso en donde se oxida y produce agua (Lodish et al., 2004); sin embargo, aunque es claro en qué es utilizado el Cu por las bacterias, aún no ha sido dilucidado para este estudio, por qué aumenta su disponibilidad a los 29 días y disminuye nuevamente a los 45 días.

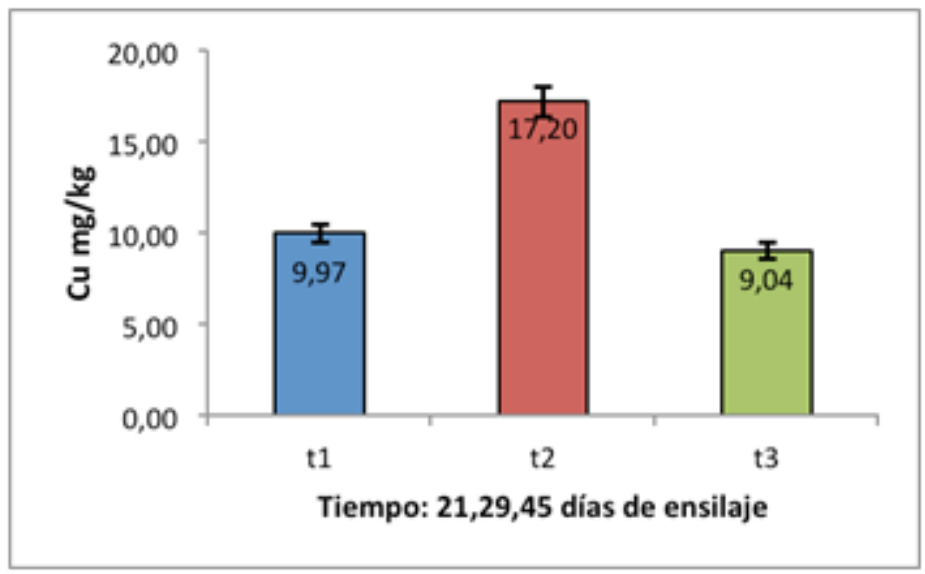

Figura 3. Comportamiento del Cu en diferentes tiempos de ensilaje.

La significancia entre los momentos fue de $\mathrm{P}=0,0388$ (0,0205 entre momentos) y Tukey indica que t1 y t3 son iguales entre sí, pero, diferentes de t2.

\section{Conclusiones}

Es factible ensilar FEN en campo, demostrando en este estudio que entre los 21 y los 45 días de fermentación, presentan características óptimas para consumo animal, sin 
embargo, debe tenerse en cuenta que la disponibilidad de algunos elementos como el Ca y el Cu presentaron variación dependiendo del tiempo de ensilaje. Los FEN ensilados se caracterizaron por su alto contenido de energía y humedad, y bajos niveles de proteína que deben ser balanceados para suplir racionalmente los requerimientos específicos de las dietas de los animales. El ensilaje de FEN ofrece una nueva estrategia para el manejo y utilización de RAC, que no genera efluentes contaminantes ni permiten el lixiviado de los nutrientes, además, es de fácil implementación en campo.

\section{Agradecimientos}

Agradecimientos a CitriCaldas y a la Gobernación de Caldas por los recursos asignados para la realización del trabajo de grado de los dos primeros autores y a la Universidad de Caldas por la disponibilidad de la granja experimental (La Cruz).

\section{Referencias bibliograficas}

- ACCPC. Acuerdo de Competitividad de la Cadena Productiva de los Cítricos.Colección documentos IICA, serie competitividad No.19, 2000.

- Aguilar, P.; Escobar, M. \& Pássaro, C. Situación actual de la cadena de cítricos en Colombia: limitantes y perspectivas. Pp 7-47. En: Garcés, L. \& Pássaro, C. (eds). Cítricos: cultivo, cosecha e industrialización. Corporación Universitaria Lasallista, editorial Artes y Letras S.A.S. Itagüí, 2012.

- AOAC. Official methods of analysis of AOAC international 19th edition, 2012.

- Bampidis, V. \& Robinson, P. Citrus by-products as ruminant feeds: A review.Animal Feed Science and Technology, v.128, p.75-217, 2006.

- Caparra, P.; Foti, F.; Scerra, M.; et al. Solar-dried citrus pulp as an alternative energy source in lamb diets: Effects on growth and carcass and meat quality. SmallRuminant Research, v.68, p.303-311. 2007.

- DANE. Encuesta Nacional Agropecuaria. Consulta: febrero de 2015. http://www.dane.gov.co/files/investigaciones/agropecuario/ena/boletin_ena_2012.pdf. 2013.

- Driehuis, F.; Oude Elferink, S. \& Spoelstra, S. Anaerobic lactic acid degradation during ensilage of whole crop maize inoculated with Lactobacillus buchneri inhibits yeast 
growth and improves aerobic stability. Journal of Applied Microbiology, v.87, n.4, p.583-594, 1999.

- Driehuis, F. \& Oude, E. The impact of the quality of silage on animal health and food safety: a review. The veterinary quarterly, v.22, n.4, p.212-216, 2000.

- Dunière, L.; Sindou, J.; Chaucheyras-Durand, F. et al. Silage processing and strategies to prevent persistence of undesirable microorganisms. Animal Feed Science and Technology, v.182, p.1-15, 2013.

- $\quad$ Fang, X.; Zhao, X.; Zhou, W. et al. Fluorescence detection and imaging of cytosolic calcium oscillations: A comparison of four equipment setups. Progress in Natural Science, v.19, p. 479-487, 2009.

- Fisher, K. \& Phillips, C. Potential antimicrobial uses of essential oils in food: is citrus the answer? Trends in Food Science \& Technology, v.19, p. 156-164. 2008.

- Gollop, N., Zakin, V. \& Weinberg, Z. Antibacterial activity of lactic acid bacteria included in inoculants for silage and in silages treated with these inoculants. Journal of Applied Microbiology, v.98, p. 662-666. 2005.

- Hacker, G. Commentary: Is there, and should there be, apoptosis in bacteria?Microbes and infection, v.15, p. 640-644. 2013.

- Holzer, M.; Mayrhuber, E.; Danner, H. et al. The role of Lactobacillus buchneri in forage preservation. Trends in Biotechnology, v.2, n.6, p.282-287, 2003.

- Isman, M. Plant essential oils for pest and disease management. Crop Protection, v.19, p. 603-608, 2000.

- Kjeldahl, J. Neve Methode Zür Besttimmung der Stickstoffs and Organischen Körpern. Z Anal Chem, v. 22, p. 366-382. 1883.

- Lante, A. \& Tinello, F. Citrus hydrosols as useful by-products for tyrosinase inhibition. Innovative Food Science and Emerging Technologies, v.27, p.154-159, 2015.

- Lobet, E.; Letesson, J.J. \& Arnould, T. Mitochondria: A target for bacteria.Biochemical Pharmacology, v.94, p. 173-185, 2015.

- Lodish, H.; Berk, A.; Matsudaira, P. et al. Energética celular. Transporte de electrones y la generación de la fuerza protón motriz. Giovaniello, O.; Méndez, A. \& Rondinone, S. (eds). Biología celular y molecular 5ta ed., p 315-325. Editorial Médica Panamericana. New York. 2004

- Londoño, J.; Sierra, J.; Álvarez, R.; et al. Aprovechamiento de los sub-productos citrícolas. En: Garcés, L. \& Pássaro, C. (eds). Cítricos: cultivo, cosecha e 
industrialización. p. 344-367. Corporación Universitaria Lasallista, editorial Artes y Letras S.A.S. Itagüí. 2012.

- Marín, F.; Soler-Rivas, C.; Benavente-García, O. et al. By-products from different citrus processes as a source of customized functional fibres. Food Chemistry, v.100, p. 736741, 2007.

- Martínez, H.; Peña, Y. \& Espinal, C. La cadena de cítricos en Colombia una mirada global de su estructura y dinámica 1991-2005. Ministerio de Agricultura y Desarrollo Rural, Observatorio Agrocadenas Colombia. Documento de trabajo No. 107. 2005.

- Martínez, J.; Chongo, B.; Jordán, H. et al. Características nutritivas de los hollejos húmedos de naranja (Citrus sinensis cv. Valencia) mantenidos en estibas. Revista Mexicana de Ciencias Pecuarias. Téc Pecu Méx, v.46, n. 2, p. 183-193, 2008.

- Muck, R.E. Recent advances in silage microbiology. Agr. Food Sci, v.22, p. 315.2013.

- Ojeda, F.; Cáceres, O.; Montejo, I. et al. Estudio de la acción del probiótico Sorbial en los indicadores nutricionales de hollejos de naranja conservados con diferentes materiales absorbentes. Pastos y Forrajes, v.31, n. 3, p. 283-292, 2008.

- Piquer, O.; Ródenas, L.; Casado, C. et al. Whole citrus fruits as an alternative to wheat grain or citrus pulp in sheep diet: Effect on the evolution of ruminal parameters.Small Ruminant Research, v.83, p. 14-21, 2009.

- Rincón, M.; Vásquez, A. \& Padilla, F. Composición química y compuestos bioactivos de las harinas de cáscaras de naranja (Citrus sinensis), mandarina (Citrus reticulata) y toronja (Citrus paradisi) cultivadas en Venezuela. Archivos Latinoamericanos de Nutrición, v.55, p. 305-310, 2005.

- Ruiz, B. \& Flotats, X. Citrus essential oils and their influence on the anaerobic digestion process: An overview. Waste Management, v.34, p. 2063-2079. 2014.

- Trimble, W. \& Grinstein, S. TB or not TB: Calcium Regulation in Mycobacterial Survival Leading Edge Previews. Cell, v.130, p. 12-13. 2007.

- Turra, C.; De Nadai, E.; Arruda, M.; et al. Chemical Elements in Organic and Conventional Sweet Oranges. Biological Trace Element Research, v.144, p. 12891294. 2011.

- Volanis, M.; Zoiopoulos, P. \& Tzerakis K. Effects of feeding ensiled sliced oranges to lactating dairy sheep. Small Ruminant Research, v.53, p. 15-21, 2004. 
- Volanis, M.; Zoiopoulos, P.; Panagouand E. et al. Utilization of an ensiled citrus pulp mixture in the feeding of lactating dairy ewes. Small Ruminant Research, v.64, p. 190-95, 2006.

- Wang, J.; Wang J.Q.; Zhou, H. et al. Effects of addition of previously fermented juice prepared from alfalfa on fermentation quality and protein degradation of alfalfa silage.Animal Feed Science and Technology, v.151, p. 280-290, 2009.

Cómo citar: Melo-Camacho, E.P.; Bermúdez-Loaiza, J.A.; Estrada-Álvarez, J. Ensilaje de naranjas enteras (Citrus sinensis) como suplemento para alimentación de rumiantes. Revista Veterinaria y Zootecnia, v. 11, n. 1, p. 24-36. DOI: 10.17151/vetzo.2017.11.1.3 